\title{
The Role of a Bioengineered Artificial Kidney in Renal Failure
}

\author{
WILLIAM H. FISSELL, JASON KIMBALL, SHERRILL M. MACKAY, \\ ANGELA FUNKE, AND H. DAVID HUMES \\ Departments of Internal Medicine, VA Medical Center and University of Michigan, \\ Ann Arbor, Michigan, USA
}

\begin{abstract}
Renal failure continues to carry substantial burden of morbidity and mortality in both acute and chronic forms, despite advances in transplantation and dialysis. There is evidence to suggest that the kidney has metabolic, endocrine, and immune effects transcending its filtration functions, even beyond secretion of renin and erythropoietin. Our laboratory has developed experience in the tissue culture of renal parenchymal cells, and has now been able to demonstrate the metabolic activity of these cells in an extracorporeal circuit recapitulating glomerulotubular anatomy. We have observed active transport of sodium, glucose, and glutathione. We describe the design and initial preclinical testing of the bioartificial kidney, as well as future directions of our research.
\end{abstract}

KEYWORDS: bioengineering; artificial kidney; renal failure

\section{SCOPE OF THE PROBLEM OF RENAL FAILURE}

The kidney is unique among body organs in that it is the first organ for which long-term ex vivo substitutive therapy has been available. Kolff's first hemodialyzer was successfully applied to a human patient with acute renal failure in 1948, and the first successful allograft transplantation was a kidney by David Humes, John Merrill, and Joseph Murray in 1951, for which a Nobel prize was awarded. Both treatments are used today. The annual mortality for patients between 40 and 60 years of age with end-stage renal disease receiving hemodialysis is $13.3 \%$, whereas that for agematched recipients of first cadaveric renal transplant is a mere $4 \% .^{1}$ The statistics for acute renal failure are similarly grim, with overall survival unmoved from its predialysis era statistic of approximately $50 \% .^{2}$ Acute renal failure as a component of multiorgan dysfunction syndrome (MODS) carries an especially poor prognosis, with survival rates around $10 \% .{ }^{3-5}$ The problem is likely to continue to grow in magnitude, since the population of patients with end-stage renal disease (ESRD) in the United States has been increasing at a constant $8 \%$ per year, although the number of kidneys transplanted has risen at about only $3 \%$ per year. 6,7

The reasons for the markedly improved prognosis of patients transplanted with allograft kidneys cannot be explained solely by the better health of patients listed for

Address for correspondence: H. David Humes, Department of Internal Medicine, Division of Nephrology, 3914 Taubman Center, University of Michigan Health System, Ann Arbor, MI 48109-0364, USA.

dhumes@umich.edu 
transplant, since those awaiting an organ have higher mortality than those actually receiving a transplant. ${ }^{7}$ We deduce that the intact or transplanted kidney provides function to the host that intermittent hemodialysis does not.

Hemodialysis provides clearance of small molecules in blood by diffusive flow across a semipermeable membrane, and control of volume status by bulk flow of water and solutes through that membrane. These short-term effects are sufficient to abrogate the lethal acidosis, volume overload, and uremic syndromes that accompany renal failure, but do not protect the patient from the increased mortality associated with dialysis-treated renal failure in either the acute or chronic form. Thus, the metabolic, endocrine, and immune roles of the functioning kidney are candidate mechanisms for the difference in survival noted above. The dialytic clearance of glutathione, a key tripeptide in free radical scavenging and protection against oxidant stress, the negative nitrogen balance and energy loss in the clearance of peptides and amino acids in dialysate, loss of oxidative deamination and gluconeogenesis in the tubule cell, and loss of cytokine and hormone metabolic activity by the kidney each impose substantial stress upon the dialyzed patient, and as such are appropriate targets for improved renal replacement therapy.

\section{RENAL CELLS AND RENAL ROLES}

The kidney's functional unit, the nephron, provides for the elimination of wastes and toxins without the need for specific enzymes and transporters for each toxin. All but the large proteins and cellular elements in the blood are filtered; a system of cells reclaims specific filtered substances needed by the body, and allows all others to pass as urine. Teleologically, this allows each organism to cope with novel insults its genetic forebears may not have encountered.

Filtration is accomplished by the glomerulus, a tuft of capillaries supported by a basement membrane and specialized epithelial cells called podocytes. The renal proximal tubule, a hollow tube of cells surrounded by capillaries, receives the filtrate from the glomerulus and accomplishes the bulk of reclamation of salt, water, glucose, small proteins, amino acids, glutathione, and other substances. The tubule also accomplishes metabolic functions including excretion of acid as ammonia, hydroxylation of 25-OH-Vit $\mathrm{D}_{3}$, and others.

Intermittent hemodialysis is thought to replace the filtration function of the glomerulus. Our attention is drawn to duplicating the function of the proximal tubule. The transport of solutes and water is accomplished by ATP-driven electrolyte transporters in the luminal cell membrane. Reabsorption of small proteins and peptides in the filtrate stream is accomplished by membrane-bound proteases and specific amino acid transport proteins within the luminal membrane of the tubule cell. These amino acids are either used for protein and peptide synthesis in the tubule cell, or are transported into the capillaries for transport to and use by the body. The diversity and specificity of the functions of the proximal tubule cell argue against the development of an electromechanical or polymeric substitute, and thus, a number of years ago, our research group turned its attention to the isolation and culture of renal proximal tubule cells. That research has culminated in the hollow-fiber bioreactor discussed below. 


\section{DESIGN AND IMPLEMENTATION OF A HOLLOW-FIBER BIOREACTOR}

\section{Selection of Proximal Tubule Cells}

Implementation and eventual manufacture of a device based on cell therapy requires a steady and predictable supply of tissues from which cells may be isolated and cultured. Until such time as stem cells may be isolated and induced to differentiate into organ-specific cell types, cells for allotransplantation, xenotransplantation, or bioartificial organ use will need to be procured through the harvest of animal or human tissues.

Human kidneys unsuitable for cadaveric transplant have been used as a source of proximal tubule cells in our laboratory, but the potential for infectious complications looms large and mandates extensive screening and testing of each donor and each harvested kidney. There is also a limited supply of these organs since they represent a side-stream of the limited supply of organs for available for transplant. Similarly, some of the characteristics rendering them unsuitable for transplantation may adversely affect the harvest of cells from the kidneys.

There has been extensive research in xenotransplantation and bioengineering with porcine cells. Researchers developing bioartificial livers ${ }^{8-18}$ and bioartificial pancreases ${ }^{19-25}$ have achieved a degree of comfort with porcine cells. Pigs are commercially bred and available in large number, and have similar organ sizes to humans. There is considerable concern about the potential for zoonotic spread of viruses, particularly after the demonstration that porcine endogenous retroviruses can infect human cells. ${ }^{26,27}$

Although most preclinical studies have used porcine cells in preparation of clinical trials, the FDA encouraged us to carry out experiments using human cells in our bioartificial kidney to continue progress in this field. Accordingly, biocomparability studies, including metabolic, transport, and endocrine functionality have been completed between porcine and human proximal tubule cells. Human cells were obtained both from postmortem specimens as well as transplant discards. Human proximal tubule cells (HPTC) have recently been characterized in order to compare functionality between the two tissue sources. Metabolic assessment included ammonia and glutathione metabolism. Porcine proximal tubule cells (PPTC) produce $10 \pm 4 \mu \mathrm{g}$ ammonia/ $10^{6}$ cells $/ 24$ hours. Ammonia production rates were $\mathrm{pH}$ responsive in both cell types. Glutathione metabolism in the PPTC and HPTC was 4.44 and $8.16 \mathrm{mmol} / 10^{6}$ cells/hour, respectively. Glutathione metabolism was inhibited in both cell types by the addition of acivicin, a specific inhibitor of glutathione metabolism. The reabsorptive function was evaluated with glucose transport. PPTC and HPTC reabsorbed $84 \pm 15$ and $96 \pm 8 \mathrm{mg}$ of glucose/10 6 cells/day, respectively. Glucose transport in both cell types was inhibited by the addition of phlorizin, a specific inhibitor of glucose reabsorption. Endocrine function was assessed by $1-25$ dihydroxyvitamin $D_{3}$ production. With the addition of parathyroid hormone, PPTC and HPTC converted $37.5 \pm 2.1$ and $48.2 \pm 5.2 \mathrm{pg} / 10^{6}$ cells $/ 24$ hours, respectively. Cytokine production was measured in both cell types. Interleukin-8 secretion was $4.7 \pm 1.7$ and $10.2 \mathrm{ng} / 10^{6}$ cells/24 hours, for PPTC and HPTC respectively, when cells were stimulated with endotoxin. These data suggest that both PPTC and HPTC 
are biocomparable with respect to transport, metabolic, and endocrine functions in cell culture.

\section{Isolation and Culture of Proximal Tubule Cells}

Our laboratory developed experience in the isolation and culture of porcine proximal tubule cells until we could reproducibly harvest cells and maintain them stably in culture. ${ }^{28,29}$ In brief, Yorkshire breed pigs were sacrificed at four to six weeks of age and their kidneys harvested. The renal cortices were dissected, minced, digested with collagenase, and the resulting mixture was separated on a Percoll density gradient. Renal proximal tubule fragments were isolated and grown in a serum-free, hormonally derived medium. After third passage and reaching confluence on $100-\mathrm{mm}$ culture dishes, cells were mobilized with trypsin into a suspension and seeded into polysulfone single hollow-fiber bioreactors (Fresenius AG BadHamburg, Germany, or Minntech, Inc., Minneapolis, MN, USA) for in vitro assessment of cell viability and metabolic activity.

\section{Characterization of a Single Hollow-Fiber Bioreactor}

Cellular attachment, stability, and confluence on the interior lumen of the bioreactor is of paramount importance. To promote attachment of the cells, the luminal surface of the polysulfone membrane was coated with ProNectin-L (Protein Polymer, San Diego, CA, USA). ProNectin-L is a synthetic protein sharing the intercellular attachment domains of laminin, a protein found in the renal glomerular and tubular basement membrane. ${ }^{30}$ Laminin and collagen type IV, key components of the tubular basement membrane, also provide an effective biomatrix for cell attachment and growth. After seeding of the hollow fiber with tubule cells, the hollow fibers were perfused with culture media. Because newly seeded cells need time to attach, perfusion was initially performed via diffusion from the exterior through the polysulfone membrane, and after time for attachment, convective flow through the interior of the fiber was initiated. A graduated increase in flow (and, thus, shear forces) was used to condition the cells and minimize cellular detachment. Studies demonstrated that confluence was reached in seven to ten days. After fourteen days in culture the hollow fiber bioreactors were assessed for cellular confluency and viability. Light microscopy of fixed sections showed evidence of a confluent monolayer formed on the inside of the hollow fibers. ${ }^{31}$

Experiments were undertaken to verify the confluency of the monolayer of cultured cells. $\mathrm{C}^{14}$-labelled inulin, a compound freely filtered by the glomerulus and by dialysis membranes, but not absorbed nor secreted by the tubule, was perfused through the lumen of the bioreactor. The amount of radioactivity in the luminal fluid and in the extraluminal fluid were compared, to verify minimal insulin transport across the monolayer, with less than two percent leak across the monolayer. ${ }^{32}$

\section{Transport and Metabolic Characteristics of Hollow-Fiber Bioreactors}

Because initial experiments using the single hollow-fiber model were promising, the design was scaled up to use commercially available polysulfone hollow fiber dialysis cartridges from the manufacturers of the single hollow fibers. Single hollow- 
fiber measurements of transport and metabolic activity were repeated with $97 \mathrm{~cm}^{2}$ and $0.4 \mathrm{~m}^{2}$ surface area cartridges.

Vectorial transport of sodium and water across the monolayers was assessed by perfusion of the lumen and the extraluminal space (ECS) with calibrated flows of culture media and measurement of timed collections of media from each flow. The bioreactor was tested to see if transport could be increased by creating an oncotic driving force across the monolayer, and if that increase could be abrogated by ouabain, a specific inhibitor of $\mathrm{Na}^{+} / \mathrm{K}^{+}$ATPase, the enzyme responsible for the bulk of sodium transport across the luminal surface of the cell, which suggested that the transport was an active process, rather than passive flow governed by Starling forces across a semipermeable membrane. To increase the oncotic pressure, bovine serum albumin (BSA) was added to the ECS perfusate, and then ouabain was also added to the ECS perfusate. The data are summarized in TABLE 1. As expected, the transport was active and mediated by sodium-potassium ATPase. ${ }^{31}$

We further explored the metabolic characteristics of the cultured proximal tubule cells. We examined the transport of glucose, bicarbonate, and glutathione and expressed the data in terms of fractional reabsorption accomplished by the bioreactor, now termed the renal tubule assist device, or RAD. For each of the molecules listed, fractional excretion was measured in the absence and presence of a known inhibitor of an enzyme essential for the reabsorption. In the case of glucose, the compound phlorizin was used; for bicarbonate, a carbonic anhydrase inhibitor, acetazolamide, was used; for glutathione, an inhibitor of $\gamma$-glutamyltranspeptidase,

TABle 1. Fluid transport ${ }^{a}$

\begin{tabular}{lcc}
\hline Ouabain Experiments & $\begin{array}{c}\text { Absolute Reabsorption } \\
(\mathrm{ml} / 30 \mathrm{~min})\end{array}$ & $\begin{array}{c}\text { Inulin Leak } \\
\text { (percent) }\end{array}$ \\
\hline Baseline & $1.05 \pm 0.40$ & $3.25 \pm 0.21$ \\
Albumin & $4.87 \pm 0.66$ & $3.10 \pm 0.21$ \\
Albumin and ouabain & $1.73 \pm 0.43$ & $2.98 \pm 0.19$ \\
\hline
\end{tabular}

${ }^{a}$ Adapted from Reference 31 .

TABLE 2. Solute transport ${ }^{a}$

\begin{tabular}{lcc}
\hline Molecule & Condition & Fractional Reabsorption (percent) \\
\hline Glucose & baseline & $25.0 \pm 1.2$ \\
Bicarbonate & phlorizin & $9.2 \pm 0.7$ \\
& baseline & $21.9 \pm 1.8$ \\
Glutathione & acetazolamide & $4.1 \pm 0.5$ \\
& baseline & $44 \pm 2$ \\
& acivicin & $25 \pm 4$ \\
\hline
\end{tabular}

${ }^{a}$ Adapted from Reference 31. 


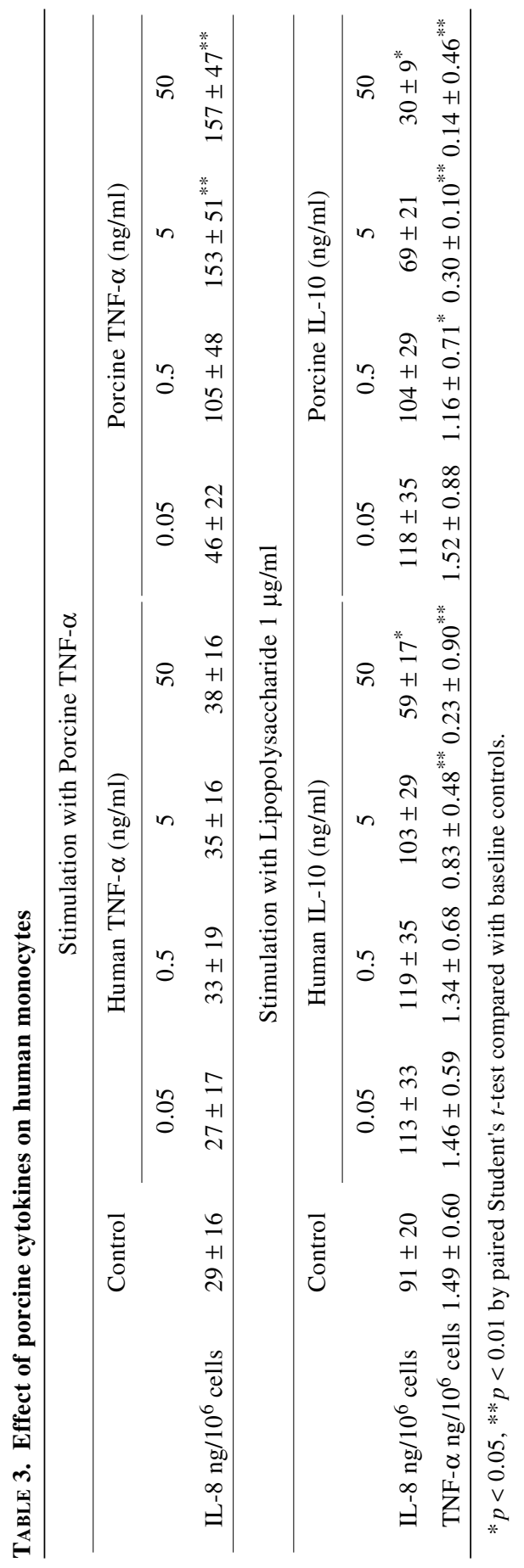


acivicin, was used. The data are summarized in TABLE 2 . In each case, there was evidence of active transport and specific inhibition. ${ }^{31}$

The synthesis and secretion of ammonia into the tubule is essential for renal excretion of an acid load, since it buffers secreted protons. Proximal tubule cells are able to upregulate their ammoniagenesis in response to a decline in $\mathrm{pH}$, and the proximal tubule cells in the bioreactor demonstrated a stepwise increase in ammonia production with changes in $\mathrm{pH}^{31}$

The experiments detailed above were performed with porcine tubule cells. However, our laboratory has demonstrated similar results in culture, attachment, and activity with human proximal tubule cells from cadaveric organs. The final selection of cell type for use in a renal tubule device rests, not only on supply and safety of cells, but also on the ability of xenotransplanted cells to participate in the homeostasis of the host. The bioequivalence of large proteins and signalling molecules from pigs and humans is not well established in the literature to date. Our laboratory undertook a series of experiments to explore this issue. Human monocytes were isolated from healthy controls by standard methods. ${ }^{33}$ Human and porcine TNF- $\alpha$ were used to stimulate monocyte production of IL- 8 in vitro. Similarly, porcine IL-10 was used to attenuate monocyte secretion of TNF- $\alpha$ and IL- 8 response to lipopolysaccharide stimulation. The results of our experiments are summarized in TABLE $3 .{ }^{34}$

This demonstration of response of human monocytes to porcine-derived cytokines is encouraging for xenotransplantation research. Of special interest is the finding that porcine IL-10 seems to be able to blunt inflammatory cytokine release from human monocytes.

The field of xenotransplantation, we must note, although a promising area of research, is challenged by much more than the aforementioned issue of bioequivalence of signals between organ and host. Detailed understandings of interactions between MHC-incompatible organs, the immune cells they harbor, and the host, as well as of zoonotic transmission in both directions are necessary before xenotransplantation research can move from laboratory to clinic. ${ }^{35,36}$

The above data suggest that our laboratory has successfully isolated and cultured renal proximal tubule cells, established stable confluent monolayers within hollow fiber bioreactors, and scaled the initial construct to a level approximating the number of proximal tubule cells in a single kidney. We termed the large-scale bioreactor the renal assist device, or RAD.

\section{EX VIVO AND PRECLINICAL CHARACTERIZATION OF THE RENAL ASSIST DEVICE}

\section{Experimental Model}

Following the demonstration of in vitro metabolic activity, we sought to determine if the metabolic and transport functions we observed in the RAD could be observed in an ex vivo system. Nephrectomized uremic mongrel dogs were placed on a modified continuous venovenous hemofiltration $(\mathrm{CVVH})$ circuit. In an analogue to the glomerular/tubule relationship seen in the kidney, ultrafiltrate from a conventional hollow-fiber dialyzer (Fresenius AG Bad Hamburg, Germany, Model F-40) was directed to the luminal space of the RAD, and a portion of the posthemofilter 


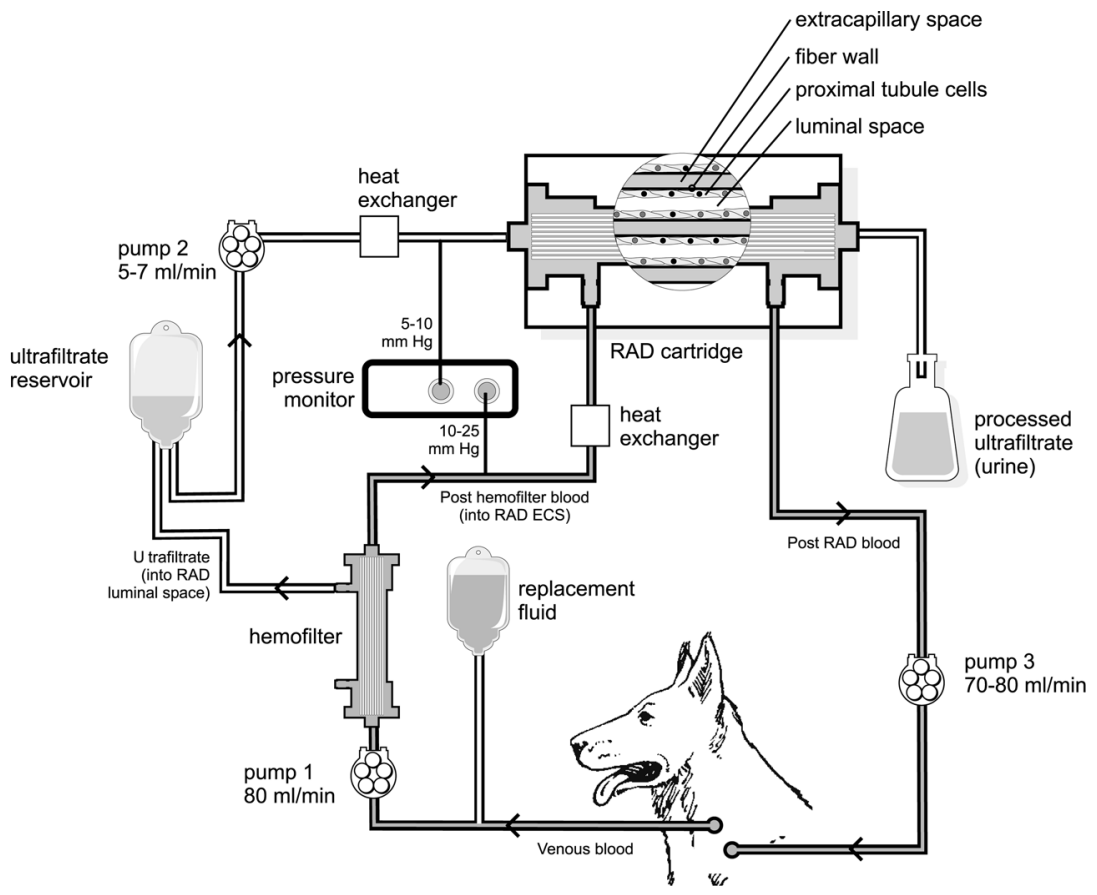

FIGURE 1. Extracorporeal hemoperfusion circuit.

blood was directed to the extracapillary space of the RAD. Roller pumps were used to dictate the flow rates of blood and ultrafiltrate to the RAD, as illustrated in FIGURE 1 . The proximal tubule is responsible for reabsorption of approximately $50 \%$ of gloneulus ultrafiltrate, and adjustment of the flow rates and pressures in the RAD allowed us to mimic that reabsorption rate in our extracorporeal perfusion circuit. A series of experiments with RADs seeded with proximal tubules and equivalent hollow-fiber cartridges without cells were conducted. To quantify cell viability, cell counts were performed on the urine leaving the RAD hourly during the RAD treatment. After removal from the circuit at the conclusion of the experiment, RAD cartridges were assessed for cell viability and confluence immediately and after return to in vitro perfusion with culture media. We examined the ultrafiltrate entering (tubular fluid) and leaving (urine) the RAD for evidence of vectorial transport and secretion by the cells. Plasma levels of electrolytes, Vitamin $\mathrm{D}_{3}$, and glutathione were measured before nephrectomy, before RAD treatment, and after RAD treatment.

\section{Cell Viability and Confluence}

Urine cell counts obtained during the treatment revealed low levels of detachment during the first hour of treatment, and little or no detachment thereafter. ${ }^{37}$ Histologic sections revealed persistence of the monolayer. Inulin leak rates increased from $5.8 \% \pm 0.9 \%$ before the experiment to $10.0 \% \pm 0.9 \%$ after the experiment, but after 
14 days of culture had improved to $6.1 \% \pm 0.6 \%$, suggesting that the cells were able to replicate and reline the interior of the lumen.

\section{Transport Functions of the RAD}

We chose to measure the homeostatic properties of the RAD not only by assessing serum concentrations of biologically important compounds, but also by quantifying vectorial transport from the ultrafiltrate to the bloodstream. The ratio of concentrations between tubular fluid leaving the RAD and ultrafiltrate entering the $\mathrm{RAD}(\mathrm{TF} / \mathrm{UF})$ is a convenient expression of vectorial transport, as it quantifies extraction of a compound from the ultrafiltrate stream. Sodium and water are transported isoosmotically by the proximal tubule in the kidney and in the RAD. TF/UF ratios for sodium were approximately 1.00 in our circuit, as expected. ${ }^{37}$ Fractional excretion of sodium did reflect active transport across the RAD, which was measured at $66.5 \% \pm 2.0 \%$ in RADs with tubule cells and $52.5 \% \pm 1.4 \%$ in sham-treated controls. ${ }^{37} \mathrm{TF} / \mathrm{UF}$ ratios were calculated for the sodium, potassium, chloride, bicarbonate, BUN, creatinine, glucose, and glutathione, and are shown in TABLE $4 .{ }^{37}$

We assessed two other measures of tubule cell metabolism: ammoniagenesis and serum 1-25 dihydroxy-Vitamin $\mathrm{D}_{3}$ levels in RAD-treated and sham-treated animals. Total ammonia excretion was assessed by collection of tubular fluid from the RAD. Excretion of ammonia was measured at $-1.38 \pm 1.2 \mu \mathrm{mol} / \mathrm{h}$ in sham-treated animals, and at $41 \pm 19.9 \mu \mathrm{mol} / \mathrm{h},(p<0.02)$ in treated animals, with peak levels exceeding $90 \mu \mathrm{mol} / \mathrm{h}$ in animals treated for 20-24 hours. ${ }^{37}$

Serum 1-25 dihydroxy-Vitamin $\mathrm{D}_{3}$ levels in animals treated with the RAD were also significantly different from levels in sham-treated controls, with levels decreasing by $4.0 \mathrm{pmol} / \mathrm{ml}$ over the course of a sham treatment, compared with an increase of $5.8 \mathrm{pmol} / \mathrm{ml}$ in RAD-treated animals. ${ }^{37}$

We have evidence that cell therapy with proximal tubule cells in our RAD bioreactor, in both in vitro and ex vivo models can reproduce many of the key functions of the renal proximal tubule. Furthermore, in large mammal models, we have demonstrated significant endocrinologic and metabolic activity reflected in serum

TABLE 4. Tubular fluid/ultrafiltrate ratios $(\mathrm{TF} / \mathrm{UF})^{a}$

\begin{tabular}{lccc}
\hline Molecule & Sham Treated & RAD Treated & $p$ \\
\hline sodium & $1.01 \pm 0.01$ & $1.00 \pm 0.01$ & NS \\
potassium & $1.00 \pm 0.02$ & $0.94 \pm 0.02$ & $p<0.001$ \\
chloride & $1.01 \pm 0.01$ & $1.00 \pm 0.01$ & $\mathrm{NS}$ \\
bicarbonate & $0.98 \pm 0.01$ & $0.96 \pm 0.01$ & $p<0.05$ \\
BUN & $1.00 \pm 0.01$ & $0.99 \pm 0.01$ & $\mathrm{NS}$ \\
creatinine & $1.00 \pm 0.01$ & $0.99 \pm 0.01$ & $\mathrm{NS}$ \\
glucose & $0.98 \pm 0.01$ & $0.88 \pm 0.04$ & $p<0.01$ \\
glutathione & $0.99 \pm 0.07$ & $0.52 \pm 0.06$ & $p<0.001$ \\
\hline
\end{tabular}

${ }^{a}$ Adapted from Reference 37. 
chemistries of the treated animal. We then directed our attention to answering one of the initial questions posed: can functioning proximal tubule cells protect against the increased mortality seen when acute renal failure complicates other illness?

\section{Role of the RAD in Animal Models in Sepsis}

Charles Natanson and others at the National Institutes of Health have developed and refined a large animal model of septic shock, using lipopolysaccharide infusions to trigger the proinflammatory cascade that characterizes the sepsis syndrome. ${ }^{38} \mathrm{We}$ adapted that model to healthy and to bilaterally nephrectomized mongrel dogs to simulate acute renal failure. Of note, even healthy dogs subject to the stress of lipopolysaccharide infusions displayed evidence of oliguric renal failure. Preliminary data suggest that some of the clinical markers of septic shock, such as hypotension, as well as serum levels of cytokines, such as IL-10, are different between dogs treated with the RAD and treated with sham controls. Further exploration is underway in defining our model.

\section{CLINICAL TRIALS OF THE RAD}

The accumulation of several years' experience with the RAD in large animal models, as well as the encouraging data from our models of septic shock have brought us to clinical trials in human patients. The FDA has given approval to proceed to a PhaseI/II clinical trial of the RAD containing human rather than porcine cells. We plan to enroll ten patients during the first half of 2001. Our inclusion criteria are designed to enroll patients with acute tubular necrosis already receiving continuous renal replacement therapy, with APACHE-III predicted mortalities between $50 \%$ and $90 \%$. We eagerly look forward to ushering in a new era of treatment for patients with acute and chronic renal failure.

\section{REFERENCES}

1. Wolfe, R., V. Ashby, E. Milford, et al. 1999. Comparison of mortality in all patients on dialysis, patients on dialysis awaiting transplantation, and recipients of a first cadaveric transplant. N. Engl. J. Med. 341: 1725-1730.

2. Alkhunaizi, A.M. \& R.W. Schrier. 1996. Management of acute renal failure: new perspectives. AJKD 28: 315-328.

3. Bone, R.C., C.J. Fischer, T.P. Clemmer, et al. 1987. A controlled clinical trial of high-dose methylprednisolone in the treatment of severe sepsis and septic shock. N. Engl. J. Med. 317(11): 653-658.

4. Davies, M.G. \& P.-O. Hagen. 1997. Systemic inflammatory response syndrome. Br, J. Surg. 84(7): 920-935.

5. Groeneveld, A., D. Tran, J. van der Meulen, et al. 1991. Acute renal failure in the medical intensive care unit: predisposing, complicating factors and outcome. Nephron 59: 602-610.

6. Haraharan, S., C. Johnson, B. Bresnahan, et al. 2000. Improved graft survival after renal transplantation in the United States, 1988 to 1996. N. Engl. J. Med. 342: 605612.

7. Neilson, E., A. Hull, J. Wish, et al. 1997. The ad hoc committee report on estimating the future workforce and training requirements for nephrology. J. Am. Soc. Nephrol. 8(5 Suppl. 9): S1-S4. 
8. Ohshima, N., K. Yanagi \& H. Miyoshi. 1999. Development of a packed-bed type bioartificial liver: tissue engineering approach. Transplant. Proc. 31: 2016-2017.

9. Busse, B. \& J. Gerlach. 1999. Bioreactors for hybrid liver support: historical aspects and novel designs. Ann. N.Y. Acad. Sci. 875: 326-339.

10. Dixit, V. \& G. Gitnick. 1998. The bioartificial liver: state-of-the-art. Eur. J. Surg. Suppl. 582: 71-76.

11. Narue, K., I. Nagashima, Y. Sakai, et al. 1998. Efficacy of a bioreactor filled with porcine hepatocytes immobilized on nonwoven fabric for ex vivo direct hemoperfusion treatment of liver failure in dogs. Artificial Organs 22: 1031-1037.

12. Naka, S., K. TAKeshita, T. Yamamoto, et al. 1999. Bioartificial liver support system using porcine hepatocytes entrapped in a three-dimensional hollow fiber module with collagen gel: an evaluation in the swine acute liver failure model. Artificial Organs 23: 822-828.

13. McLaughlin, B., C. Tosone, L. Custer, et al. 1999. Overview of extracorporeal liver support systems and clinical results. Ann. N.Y. Acad. Sci. 875: 310-325.

14. Patzer, J., G. MaZariegos, R. Lopez, et al. 1999. Novel bioartificial liver support system: preclinical evaluation. Ann. N.Y. Acad. Sci. 875: 340-352.

15. Roger, V., P. Balladur, J. Honiger, et al. 1998. Internal bioartificial liver with xenogeneic hepatocytes prevents death from acute liver failure: an experimental study. Ann. Surg. 228: 1-7.

16. IwATA, H., T. SAJIKI, H. MAEDA, et al. 1999. In vitro evaluation of metabolic functions of a bioartificial liver. ASAIO J. 45: 299-306.

17. Watanabe, F., C. Mullon, W. Hewitt, et al. 1997. Clinical experience with a bioartificial liver in the treatment of severe liver failure. a phase I clinical trial. Ann. Surg. 225: 484-491.

18. Watanabe, F., C. Shackleton, S. Cohen, et al. 1997. Treatment of acetaminopheninduced fulminant hepatic failure with a bioartificial liver. Transplant. Proc. 29: 487-488.

19. Hayashi, H., K. Inoue, T. Aung, et al. 1996. Long survival of xenografted bioartificial pancreas with a mesh-reinforced polyvinyl alcohol hydrogel bag employing a b-cell line (min6). Transplant. Proc. 28: 1428-1429.

20. Delaunay, C., S. Darquy, J. Honiger, et al. 1998. Glucose-insulin kinetics of a bioartificial pancreas made of an an69 hydrogel hollow fiber containing porcine islets and implanted in diabetic mice. Artificial Organs 22: 291-299.

21. Calafiore, R., G. Basta, L. Osticioli, et al. 1996. Coherent microcapsules for pancreatic islet transplantation: a new approach for bioartificial pancreas. Transplant. Proc. 28: 812-813.

22. Ohgawara, H., S. Hirotani, J. Miyazaki, et al. 1998. Membrane immunoisolation of a diffusion chamber for bioartificial pancreas. Artificial Organs 22: 788-794.

23. Oberholzer, J., F. Triponez, J. Lou, et al. 1999. Clinical islet transplantation: a review. Ann. N.Y. Acad. Sci. 875: 189-199.

24. Hunter, S., Y. WANG, C. WeINER, et al. 1997. Encapsulated beta-islet cells as a bioartificial pancreas to treat insulin-dependent diabetes during pregnancy. Am. J. Obstet. Gynecol. 177: 746-752.

25. Hunter, S., Y. Wang \& V. Rodgers. 1999. Bioartificial pancreas use in diabetic pregnancy. ASAIO J. 45: 13-17.

26. Martin, U., V. Kiessig, J. BlusCh, et al. 1998. Expression of pig endogenous retrovirus by primary porcine endothelial cells and infection of human cells. Lancet 352: 692-694.

27. Patience, C., Y. Takeuchi \& R. Weiss. 1997. Infection of human cells by an endogenous retrovirus of pigs. Nat. Med. 3: 282-286.

28. Humes, H. \& D. Cieslinski. 1992. Interaction between growth factors and retinoic acid in the induction of kidney tubulogenesis in tissue culture. Exp. Cell Res. 201: $8-15$.

29. Humes, H., J. Krauss \& D. Cieslinski, et al. 1996. Tubulogenesis from isolated single cells of adult mammalian kidney: clonal analysis with a recombinant retrovirus. Am. J. Physiol. 271: F42-49. 
30. Ogawa, S., Z. Ota, K. Shikata, et al. 1999. High-resolution ultrastructural comparison of renal glomerular and tubular basement membranes. Am. J. Nephrol. 19: 686693.

31. Humes, H., S. MacKay, A. Funke, et al. 1999. Tissue engineering of a bioartificial renal tubule assist device: in vitro transport and metabolic characteristics. Kidney Int. 55: 2502-2514.

32. MackAy, S., A. Funke, et al. 1998. Tissue engineering of a bioartificial renal tubule. ASAIO J. 44: 179-183.

33. BoyUM, A. 1998. Isolation of mononuclear cells and granulocytes from human blood. Scand. J. Clin. Lab. Invest. 21: 77.

34. Kimball, J., A. Funke, D. Buffington, et al. 2000. Effect of porcine cytokines on human peripheral blood mononuclear cells. Abstract. J. Am. Soc. Nephrol. 11: $665 \mathrm{~A}$.

35. Cozzi, E., S. Masroor, B. Soin, et al. 2000. Progress in xenotransplantation. Clin. Nephrol. 53: 13-18.

36. Goddard, M., J. Foweraker \& J. Wallwork. 2000. Xenotransplantation-2000. J. Clin. Pathol. 53: 44-48.

37. Humes, H., D. Buffington, S. MacKay, et al. 1999. Replacement of renal function in uremic animals with a tissue-engineered kidney. Nat. Biotech. 17: 451-455.

38. Natanson, C., P. Elchenholz, R. Danner, et al. 1989. Endotoxin and tumor necrosis factor challenges in dogs simulate the cardiovascular profile of human septic shock. J. Exp. Med. 169: 823-832. 